\title{
Ausbildung und Beruf
}

\section{Eine Wende}

\section{in der Laboratoriumsmedizin?}

Von Dr. med. Hermann Lommel

Vorsitzender der Deutschen Gesellschaft für Laboratoriumsmedizin

zugleich Arbeitsgemeinschaft der Fachärzte für Laboratoriumsmedizin e. V.

$\mathrm{F}$ alls die jetzige Regierungskoalition noch mehrere Jahre die Entscheidungen in der Gesundheitspolitik der Bundesrepublik fällt, wird die Zeit nicht mehr fern sein, in der auch die ambulante kassenärztliche Versorgung durch angestellte Ärzte erfolgt. Der niedergelassene Kassenarzt ist dann kein Angehöriger eines freien Berufes mehr, zumindest nicht mehr auf der Seite seiner Rechte. Auf der Gegenseite des. Gleichgewichtes, auf der Seite seiner Pflichten dagegen, mag die Waagschale noch reichlich gefüllt sein. Wie dies im einzelnen auch aussehen würde, sei dahingestellt, die ersten Schienen in diese Richtung sind bereits gelegt. Entweder sind es vorsorglich gelegte 'Geleise, deren Richtung durch spätere kleine Weichenstellungen schlagartig erkennbar wird, oder es sind bereits Charakteristika aus den Angestellten-Berufen, die schon jetzt wie Fremdkörper im bisherigen System auffallen.

Der Bedarf der kassenärztlichen Versorgung muß bis ins Einzelne geplant werden [\$ 368 (4) und $\S 368$ p (7) RVO], die Richtlinien für die Bedarfsplanung sind bereits seit Juli 1977 erarbeitet. Hiernach gibt es Planungsbereiche für die allgemeinärztliche und für die fachärztliche Versorgung, es sollen Übersichten regelmäßig über den Stand der kassenärztlichen Versorgung erstellt werden, die Bedarfsermittlung erfolgt über Meß- zahlen aus Einwohnerzahlen und Kassenarztzahlen, so zum Bei- . spiel ein Allgemeinarzt auf 2400 Einwohner, ein Internist auf 10000 , ein Kinderarzt auf 25000 , ein Urologe auf 66000 , ein Laborarzt findet sich hier nicht. Es werden Planungsblätter angelegt, die Versorgung laufend statistisch verfolgt und ein Warnsystem aufgebaut zur rechtzeitigen Erkennung einer drohenden Unterversorgung. Arztstrukturdaten ermöglichen eine Gruppierung nach Fachbezeichnung, Zahl der Abrechnungsfälle im kurativen und im präventiven Bereich, örtlicher Zugehörigkeit, zusätzlichen ärztlichen Tätigkeiten, Art der Praxisausübung und vielem mehr - alles wie in einem Stellenplan.

Aufgrund des KVKG müssen bei der Veränderung der Gesamtvergütungen unter anderem ,die zu erwartende Entwicklung ... der für kassenärztliche Tätigkeit aufzuwendenden Arbeitszeit sowie Art und Umfang der ärztlichen Leistungen, soweit sie auf einer gesetzlichen oder satzungsmäßigen Leistungsausweitung beruhen", berücksichtigt werden [\$368f (3) RVO]. Ferner muß der niedergelassene Kassenarzt die Beendigung seiner Tätigkeit mit Frist kündigen [\$ $368 \mathrm{c}(2) \mathrm{RVO}]$.

Die kassenärztliche Versorgung wird nicht mehr nach dem Prinzip einer Versicherung mit Einfluß von. Risiko und anfallenden Lei- stungen, vielmehr nach dem einer Versorgung mit Vorausschätzung der Wirtschaftsentwicklung aufgebaut, die Gesamtvergütung wird zentral gesteuert, ist mehr oder minder festgelegt (,,eine Betondecke eingezogen"), und auch für Arzneimittel ist ein Höchstbetrag ,zu bestimmen“ [ $\$ 368 \mathrm{f}(1)-(7)$ RVO]. Für alle ärztlichen Leistungen für alle Krankenkassen wird ein ,einheitlicher Bewertungsmaßstab" vereinbart [\$368g (4) RVO].

In Hamburg wurde der (fehlgeschlagene) Versuch eines Gesundheitssystems unternommen, der Ärztekammer ausschließlich die niedergelassenen Ärzte als Zwangsmitglieder zuzuorỏnen, Krankenhausärzte und andere aber nicht. Soll die Kammer der Vorläufer des ersten „Arbeitgebers" sein? Eine Arbeitnehmermentalität unter den Ärzten wird durch die zunehmende Verschulung des Medizinstudiums ohnehin gefördert.

Das Bundesforschungsministerium soll durch ein Forschungsvorhaben das Ziel einer vollständig anderen Ausbildung in der Art einer medizinischen Gesamtschule mit dem Endziel „Arzt als Arbeitnehmer" anstreben. Und sollte der ,angestellte niedergelassene Arzt" den Achtstundentag noch nicht einhalten können, so werden ihm spätestens 198315000 arbeitslose Ärzte bei seiner Zeiteinteilung be- 
hillich scin. V'on der Bundesregicrung sollen 750)( Studienanfäinger jührlich in der Medizin ingestrebt werden. so dab in 20 Jahren cin Arat etwa 340 Binwolnner versorgt (gegenwärtig 500 Einwoliner).

Die gesundheitspolitischen Leitsïtze der SPD vom November 1977 wollen das Vertragsrecht durch sogenannte ,.Selbstverwallungen" crsetzen, in denen die Ärztc höchstens cin Drittel der entscheidenden Stimmen haben. Sic sollen die medizinisch-technischen Zentren, Gruppenpraxen, medizinisch-technischen Gemeindezentren, Neubau von Krankenhäusern und andere zentrale Einrichtungen steuern. Sie sollen ferner das Angebot medizinischer Leistungen vollständig an die Inanspruchnahme anpassen (Planwirtschaft?) und den Krankenkassen eine Art Vetorecht einräumen.

Was hat das alles mit . Laboratoriumsmedizin zu tun?

Geht auch hier auf dem Umweg über die „Gemeinschaftseinrichtung niedergelassener Ärzte" (GEnÄ) gemäß § $368 \mathrm{n}(8) \mathrm{RVO}$ der Weg in dieselbe Richtung, zum Beispiel über den Erwerb der GEnÄ durch die ärztlichen Körperschaften als medizinischtechnische Zentren mit angestellten Ärzten? Oder sollen auf dem Umweg über die Arbeitsmedizin entsprechend den Vorstellungen der SPD die Laboruntersuchungen im Betrieb des Arbeitnehmers veranlaßt und in ,überörtliche Zentren " weitergeleitet werden zur Ergänzung von Standardprogrammen für Basisuntersuchungen bei Diagnostik und Früherkennung? Wir wissen es nicht, aber als Wähler haben wir alle Möglichkeiten der Mitentscheidung.

Zur Zeit können und müssen wir mit den gegenwärtigen Gesetzen lehen. Sic sehen mit Wirkung vom 1. Juli 1978 u.a, vor, daß die Kassenärztlichen Vereinigungen eine Zusammenfassung und Rationalisierung von Laboratoriumsuntersuchungen ermöglichen sollen, zumindest für einen kleinen Teilbereich der Med. Chemie. Andere Bereiche erfordern entweder den Laborarzt oder mindestens einen Arzt mit ausgiebiger Teilweiterbildung in Laboratoriumsmedizin, ferner die ständige Mitarbeit der unentbehrlichen MTA und verlangen auch eine ganz andere Struktur des Laboratoriums.

Das Laborkonzept der nahen $\mathrm{Zu}$ kunft - zumindest für die Kassenpraxis - wird im wesentlichen geprägt werden durch

$\S 368 \mathrm{n}(8) \mathrm{RVO}$ : „Die Kassenärztlichèn Vereinigungen haben darauf hinzuwirken, daß medizinisch-technische Leistungen, die der Arzt zur Unterstützung seiner Maßnahmen benötigt, wirtschaftlich erbracht werden. Die Kassenärztlichen Vereinigungen sollen ermöglichen, solche Leistungen im Rahmen der kassenärztlichen Versorgung von Gemeinschaftseinrichtungen der niedergelassenen Ärzte zu beziehen, wenn eine solche Erbringung medizinischen Erfordernissen genügt."

und

$>$ die Begrenzungen der Ausgaben für Laborleistungen entsprechend den Empfehlungsvereinbarungen.

Die Kassenärzte müssen sich also mit dem Problem der Gemeinschaftseinrichtung niedergelassener Ärzte (GEn ̈̈) auseinandersetzen, so auch der Berufsverband der Laborärzte, der dies in den letzten Monaten getan und der Kassenärztlichen Bundesvereinigung seine Vorstellungen vorgetragen hat. Diese sind auch in groben Zügen am 10. Dezember 1977 der Vertreterversammlung der Kassenärztlichen Bundesvereinigung dargelegt worden*. Die

* Der volle Wortlaut wird im Februar-Heft abgedruckt.
Vorstellungen gehen von folgenden Voraussetzungen aus:

1. Auch in Gemeinschaftscinrichtungen kann ein Ar\%t nur Leistungen „,bezichen“, die seiner fachlichen Vorbildung, der Ausbildung zum Arzt, entsprechen und in einem Spektrum mit Analysenqualität festgelegt sind.

2. Die Gemeinschaftseinrichtungen müssen von einem Arzt, und zwar dauernd, beaufsichtigt werden.

3. Überschreiten sie eine bestimmte Größe oder werden teil- bzw. vollmechanisierte Systeme eingesetzt, muß dieser Arzt mindestens eine Teilweiterbildung in Laboratoriumsmedizin (1 Jahr Med. Chemie) nachweisen oder Laborarzt sein.

4. Die Entfernung zwischen Mitgliedern und GEnÄ darf nicht größer als $30 \mathrm{~km}$ sein.

5. Das begrenzte Spektrum der Untersuchungen darf in den GEnÄ nicht überschritten werden.

6. Werden Vielkanalmaschinen zur Erstellung sogenannter „Profile" eingesetzt, dann handelt es sich um einen ScreeningTest (Suchmethode), für den besondere Bedingungen bestehen; werden hierbei verdächtige oder pathologische Werte ermittelt, dürfen sie nicht in demselben System „,kontrolliert", sondern müssen durch gezielte Analysen über-. prüft werden.

7. Die Kassenärztlichen Vereinigungen müssen sicherstellen, daß ihre eigenen Richtlinien auch tatsächlich eingehalten werden.

8. Bei aller Bedeutung des im Gesetz besonders hervorgehobenen wirtschaftlichen Gesichtspunktes darf das Kaufmännische niemals das Fachmännische in der Priorität verdrängen; dies erwartet jeder Patient als eine Selbstverständlichkeit. 
Unter diesen Bedingungen ,bezogene" medizinisch-technische Leistungen (Laboratoriumsuntersuchungen) bleiben ärztliche Verrichtungen und können als solche abgerechnet werden.

Natürlich wird man bei der Organisation von .,Gemeinschaftseinrichtungen niedergelassener Ärzte" bestehende Strukturen berücksichtigen müssen. Dies sind die Laborgemeinschaften. die Laborarztpraxen.

Dies entspricht auch der Auffassung des Vorsitzenden der der FDP-Fraktion im Deutschen Bundestag (sein Brief vom 23. Mai 1977 an einen Laborarzl):

..Mit dieser Bestimmung ist den Kassenär:tlichen Vereinigungen nicht vorgeschrieben, medizinischtechnische Leistungen in Gemeinschaftseinrichtungen zu zentralisieren. Die KVen sollen es 'nur eben ermöglichen, dicse Leistungen auch durch geeignete Gemeinschaftseinrichtungen anbieten zu lassen. Die nicht in Gemeinschaftseinrichtungen arbeitenden Laborärzte sollen dadurch keinesfalls ausgeschlossen werden. Da die ganze Vorschrift unter dem Gebot der Wirtschaftlichkeit steht, haben die KVen im Gegenteil sogar darauf hin=uwirken, daß diese Leistungen von Laborärzten bezogen werden. die besonders wirtschaftlich arbeiten."

Bei der Laborgemeinschaft, mit oder ohne Mitwirkung eines Laborarztes, besitzen die Mitglieder gemeinsam die Einrichtungen und beziehen ihre medizinisch-technischen Leistungen ebenfalls gemeinsam.

Bei der Labormutzung der Einrichtungen einer Laborarztpraxis (Einzelpraxis bzw. Gemeinschaftspraxis) werden die medizinischtechnischen Leistungen gemeinsam bezogen und dem Besitzer der Einrichtung ein Nutzungsentgelt entrichtet, das nur die nachgewiesenen Unkosten für die Nutzung abdeckt. Daneben wäre ein ärztliches Honorar für die spezifische ärztliche Leistung der Methodenwahl, Sicherstellung der Organisation und Qualitätsmaßnahmen (know how') getrennt zu liquidieren.

Im Fall der Laborgemeinschaft erfolgt die externe Qualitätssicherung durch Teilnahme der Gemeinschaft an Ringversuchen mit befreiender Wirkung für alle Mitglieder, bei der Labornutzung nimmt jeder Arzt an Ringversuchen teil, schleust die Proben in die Gemeinschaftseinrichtung ein und hat damit den ungeheuren Vorteil einer echten Über-. prüfung der Richtigkeit des benutzten Systems zu jeder von ihm gewählten Zeit und bietet den höchsten Grad von Sicherheit. Auf diese Weise können - zumindest in der Med. Chemie die großen Kapazitäten bestehen- der Laboreinrichtungen voll genutzt werden und ein Teil des Rationalisicrungseffektes an den Kostenträger weitergegeben werden bei Aufrechterhaltung einer hohen Güte der Untersuchungsergebnisse. So wird in den mit Datenverarbeitung zum Teil mehr oder minder vollständig, zum Teil im kompletten On-line-System arbeitenden Laborarztpraxen die Serienlänge der gezielten Untersuchungen größer, so daß der Kostenteil für die Untersuchungen der Labornutzung auf die Hälfte bis ein Drittel absinkt (nach R. Haeckel (I) sind die Analysenkosten je Probe am Beispiel der Bestimmung der Aktivität der GOT bei einer Serienlänge von enta 10 Proben dreimal so hoch und bei ciner Serienlänge von enra 30 Proben zweimal so hoch wie bei

(1) R. Haeckel ,.Empfehlungen zur rationellen Organisation von ärztlichen Laborgemeinschaften". Deutscher Ärzte-Verlag 1976.

Die Liste der Bestandteile, die von den GEn $\ddot{\text { b bezogen }}$ werden können (Grund- und Basisuntersuchungen), lautet nach den Vorstellungen der Deutschen Gesellschaft für Laboratoriumsmedizin:

Med. Chemie

Gesamteiweiß

Albumin

Bilirubin

Glukose

Cholesterin

Triglyzeride

Natrium

Kalium

Calcium

Chlorid

Hämatologie, Gerinnung

Hämoglobin

Hämatokrit

Differentialblutbild

Retikulozytenzahl

$\begin{array}{ll}\text { Aktivitäten der } & \text { Harnstoff } \\ \text { GOT } & \text { Harnsäure } \\ \text { GPT } & \text { Kreatinin } \\ \text { Gamma-GT } & \\ \text { LAP } & \text { gegebenenfalls } \\ \text { LDH } & \text { Urinstatus } \\ \text { HBDH } & \\ \text { AP } & \text { Suchteste } \\ \text { SP } & \\ x \text {-Amylasc } & -\end{array}$

Erythrozyten-

zahl

Leukozyten-

zahl

Thrombo-

zytenzahl
Blutungszeit

Quicktest

Partielle

Thromboplastinzeil 


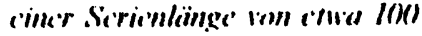
Prohen hei liruchdemg dex (ieräle's l:ppendorf 50 sill o $k$ : bei lior.

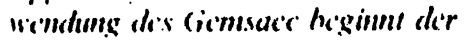

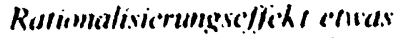
später. secigt dlamn aher he'i weiter zumihme'mder Serientënge ïher 1.5) his $2(6)$ Prohen noch weiter ani. Deshalb müssen die tatsätchlichen Inkosten für eine Labornulsung in jedem Einzelfall besonders ermitlelt werden und brauchen nicht in Zusammenhang $z u$ stchen mit der Höhe des in ciner Gebührenordnung ausgewiesenen llonorars.

$\mathrm{Ob}$ in Zukunft die Leistungen der GEn $\ddot{A}$ auf die obige Liste beschränkt bleiben, sei dahingestellt. Ob Ärztekammer und Kassenärztliche Vereinigung zu den echten Konsequenzen bereit sind und die innerärztliche Abgrenzung auf dem Gebiet der Laboratoriumsmedizin, die schon längst fällig ist und deren Ausbleiben nicht ganz unschuldig an der Entwicklung der letzten Jahre sein dürfte, tatsächlich vollziehen, sollte nicht mehr weiter dahingestellt bleiben. Die Ausbildung des Arztes, seine Weiterbildung und seine damit erworbenen Kenntnisse und Fähigkeiten müssen auch auf dem Gebiet der Laboratoriumsmedizin die erforderlichen Grenzen abstecken. Nur so kann auch der Laborarzt in Zukunft die jeweils modernen Untersuchungsverfahren aufnehmen und die zugehörigen Investitionen wagen. Er hat sich in den vergangenen Jahrzehnten als Schrittbereiter in Krankenhaus und Praxis für die in Wissenschaft und Forschung entwickelten Untersuchungsmethoden erwiesen und soll es auch in $\mathrm{Zu}$ kunft bleiben.

In der Vergangenheit waren es die Laborärzte, die in der kassenärztlichen Versorgung die serologische Mutterschaftsvorsorge, später die zytologische Krebsfrüherkennung und dann die Isotopenanwendungen im Laboratorium einführten und sicherstellten. In der Zukunft werden sie ihr Schwergewicht auf andere moderne Arbeitsgebiete legen müssen. immer an der Grenze Forschung. Fiforschung. lirfindung einerseits und Ubernahme in die tägliche Anwendung andererseits. Dies wird sich vor allem als notwendig erweisen bei den Verfahren der Immunologie - insbesondere Fluoreszenzmikroskopie und HLA-Immunologie - bei den Hormonanalysen und den zugehörigen Funktionstesten, in der Verlaufskontrolle durch Arzneimittelbestimmungen, ferner bei der Anwendung von Zellkulturen zur Chromosomendiagnostik; hier eröffnet sich ein ungeheuer segensreiches Feld für die echte Vorbeugung durch Früherkennung von Gesundheitsgefahren bzw. Krankheiten der Nachkommen schon im Mutterleib. Andere Arbeitsgebiete werden folgen. Die Deutsche Gesellschaft für Laboratoriumsmedizin wird die zugehörigen Fortbildungsveranstaltungen anbieten.

Ich halte es nicht für richtig, wenn der Wissenschaftler bei der Vorstellung neuer Verfahren vor einer breiteren ärztlichen Öffentlichkeit auf die Möglichkeit hinweist, daß diese Anwendungen nur in Speziallaboratorien oder Universitätszentren durchgeführt werden können. Universitäten und große Krankenanstalten haben ihre Zentrallaboratorien, der niedergelassene Arzt soll aber die Möglichkeit nicht verlieren, Untersuchungsverfahren, deren Anwendung in der täglichen Diagnostik sinnvoll, zweckmäßig und wirtschaftlich ist, auch in seinem. . Bereich ausführen zu lassen, also bei seinem Laborarzt. Auch der Hinweis mancher Herstellerfirmen auf besondere Spezialisten oder Zentren bei ihren Empfehlungen für bestimmte Laboratoriumsuntersuchungen wird den Gegebenheiten nicht gerecht. Der Fortschritt zieht durch alle Instanzen und sollte gefördert, nicht aber gebremst werden.

Genau wie in der Industrie wird auch gerade in der Laboratoriumsmedizin infolge des ungeheuer schnellen Erkenntniszuwachses in Biologic und Naturwissenschaften der Hauptanteil der ..Produktion“ auf junge und moderne ..Produktc" entfallen müssen. Die Firma Siemens teilt zum Beispiel mit, daß 43\% ihrer Erzeugnisse jünger als fünf Jahre sind, die Firma Agfa-Gevaert bestreitet $40 \%$, ihres Umsatzes auf dem Fachsektor mit Produkten, die erst seit zwei Jahren hergestellt werden.

Unter diesem Gesichtspunkt ständiger Weiterentwicklung und unter Berücksichtigung der Tatsache, da $B$ auch in der Laboratoriumsmedizin stets das Fachmännische vor dem Kaufmännischem den Vorrang haben muß. ist es weder richtig noch kollegial, dem Laborarzt vorzuhalten, er ginge ,mit der Gebührenordnung ins Bett". Er hat keinen Einfluß auf die Art, die Häufigkeit und den Zeitpunkt einer bestimmten Untersuchung, hat eine ,Lieferfrist" von Stunden bis Tagen und muß täglich eine große Anzahl, oft mehr als hundert verschiedene Verfahren, bereithalten. Er tut dies gerne, hat er sich doch selbst seinen Beruf gewählt. Er erwartet aber auch Gerechtigkeit in der Beurteilung seines Bemühens, den behandelnden Arzt jederzeit mit seinen Möglichkeiten zu unterstützen. Dies gilt auch ganz besonders für die Gemeinschaftseinrichtungen niedergelassener Ärzte (GEnÄ).

Um das zukünftige Volumen der gemeinschaftlichen Laboratoriumsuntersuchungen zu bewältigen, müßten die vorhandenen Einrichtungen ausreichen. Ärztekammern und Kassenärztliche Vereinigungen dürften aber auch öffentlich ruhig einmal feststellen, daß ein echter Nachwuchsbedarf auf diesem Fachgebiet besteht und daß der junge Arzt mit Interesse für Laboratoriumsmedizin nicht verunsichert werden darf. Die behandelnden Ärzte der kommenden Jahre brauchen den jungen Laborarzt mehr und mehr, und nicht etwa weniger als bisher. 
Deshalb wende ich mich mit diesen Zeilen ganz besonders an die Studenten und die jungen Ärzle mit dem Vorschlag, in den Laboratorien der Krankenhäuser bzw. den Laborarztpraxen zu famulieren und sich über die Möglichkeiten einer Weiterbildung genau zu unterrichten. Die Breite des Fachgebietes erfordert ohnehin eine gemeinschaftliche Berufsausübung durch mehrere Laborärzte, falls das gesamte Spektrum abgedeckı werden soll. Die Schaffung von Teilgebieten für Mikrobiologie und eventuell später auch andere abgrenzbare Tätigkeitsbereiche würde in Krankenhaus und Praxis zur Unterstützung der behandelnden Ärzte bei kurativen und präventiven Maßnahmen sowie zur Verlaufskontrolle und bei Funktionsprüfungen am Patienten ungewöhnlich förderlich sein. Teilgebietsbezeichnungen betonen das Gemeinsame und nutzen die vielen Möglichkeiten von Querverbindungen innerhalb der Laboratorien. Die Abtrennung eigener Facharztbezeichnungen würde zu nachteiligen Abtrennungen führen, wie sie kaum in anderen Ländern anzutreffen sind. Die Laboratoriumsmedizin (Clinical Pathology) mit der Möglichkeit der Gebietsbezeichnung „Laborarzt" nutzt dem behandelnden Arzt am meisten, wenn ihre Vertreter untereinander den Kontakt und die Querverbindungen behalten, wie sie ein gemeinsames Dach fordert.

> Konsultation (vor einer Probennahme)

$>$ Auswahl und Qualität des Untersuchungsganges

$>$ Interpretation des Untersuchungsergebnisses (= Befund)

sind die drei Säulen des Laborarztes, mit denen er dem behandelnden Arzt zur Verfügung steht. Darüber hinaus ist er bemüht, diesem auf zahlreichen Kongressen und anderen Veranstaltungen und auch mit dieser Zeitschrift die notwendige Fortbildung zu ermöglichen, damit das Gespräch nie versiegt.

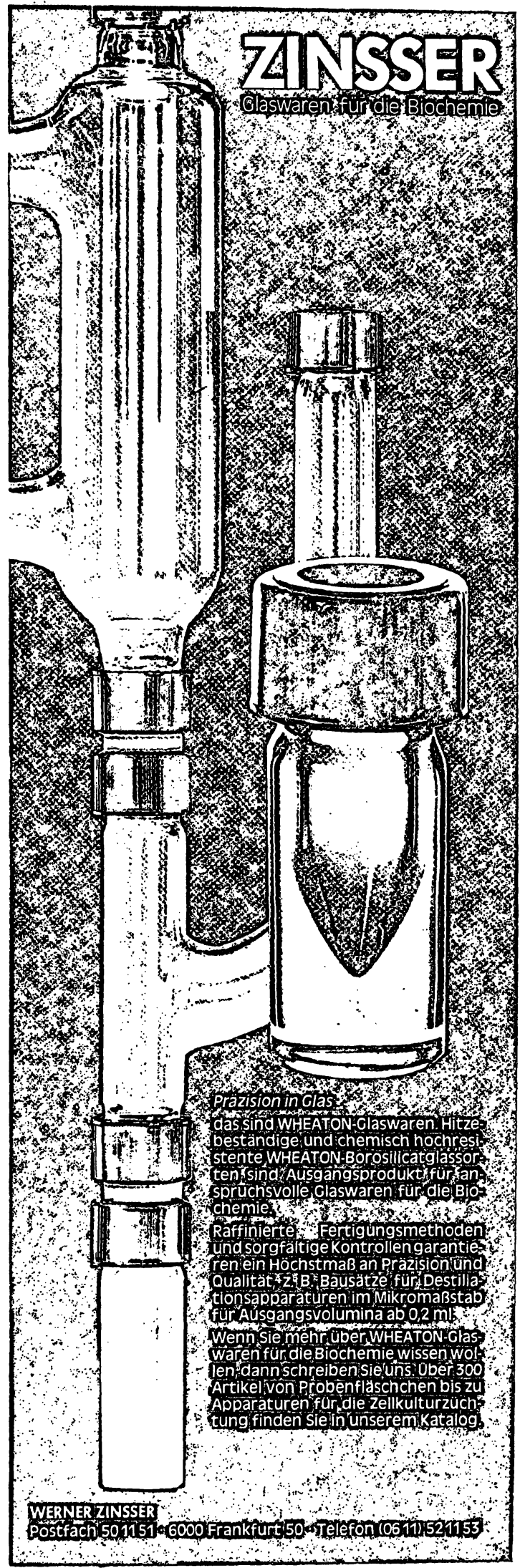


Testseren

Serologische Reagenzien von BIOTEST

\section{Qualität gibt Vertrauen}

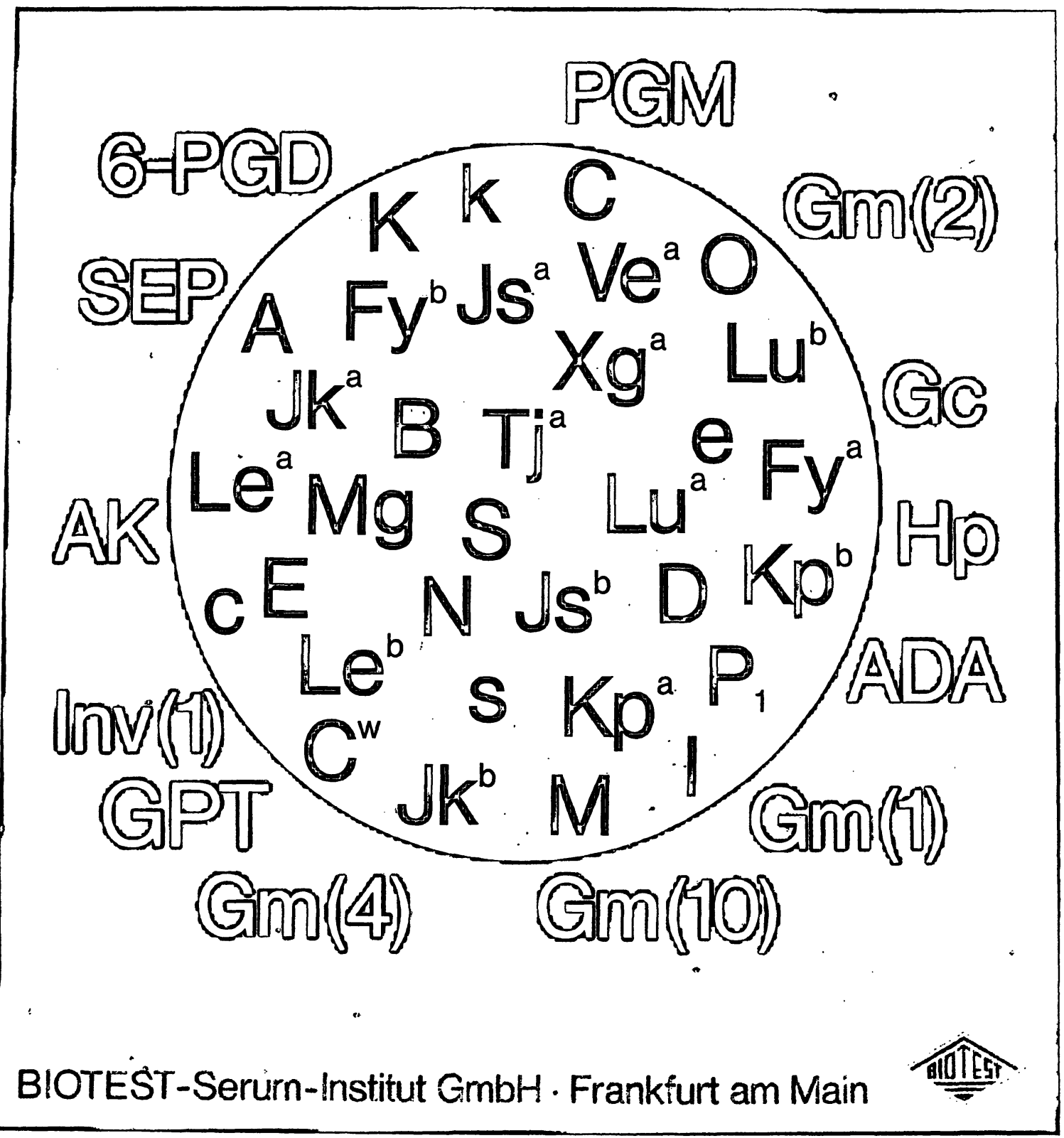




\section{Mitteilungen aus der Deutschen Gesellschadt für Laboratoriumsmedizin}

\section{Herbsttagung 1977 Aus der Arbeit der Arbeitskreise und Ausschiisse}

Im Dezember-Heft 1977 unserer Zeitschrift wurde ein erster Bericht gegeben über die Herbsttagung 1977 der Deutschen Gesellschaft für Laboratoriumsmedizin und des Berufsverbandes der Laborärzte (Arbeitsgemeinschaft der Fachärzte für Laboratoriumsmedizin). Sie fand als 11. Fortbildungsveranstaltung in Essen-Kettwig vom 13. bis 15. November 1977 statt und hatte $\mathrm{zu}$ Hauptthemen

a) Immunfluoreszenz und

b) HLA-System.

Wenn auch der wissenschaftliche Teil sehr viele Mitglieder anzog und wesentliche Informationen vermittelte, so war dies nicht minder der Fall für die beruflichen Veranstaltungen durch den Berufsverband der Laborärzte, die Arbeitsgemeinschaft der Fachärzte für Laboratoriumsmedizin in der Deutschen Gesellschaft für Laboratoriumsmedizin e. V.

In 12 getrennten Sitzungen tagten Arbeitskreise, Ausschüsse und andere Gremien. Die Mitgliederversammlung traf sich zweimal und beschäftigte sich besonders eingehend mit den $\mathrm{Zu}$ kunftsaussichten der Laboratoriumsmedizin. Unter der Überschrift „Drohen der Laboratoriumsmedizin Teikung, Aufteilung, Verteilung?"“ diskutierten die Mitglieder des Berufsverbandes der Laborärzte und verabschiedeten Resolutionen, darunter auch jene über die Gestaltung von Laborgemeinschaften, die im De-
zember-Heft auf Seite XXXI veröffentlicht wurde. Der Ausschuß „Laborgemeinschaften" (Vorsitz: Dr. med. F.-G. Weyer), in dem diese Resolution erarbeitet worden war, hatte zusätzlich folgenden einstimmigen Beschluß gefaßt:

„Der Ausschuß ,Laborgemeinschaften“ sieht eine ungleiche Behandlung zwischen Laborärzten und anderen Fachgruppen darin, daß Laboratoriumsuntersuchungen, die in dem Weiterbildungskatalog jener Fachgruppen enthalten sind, nicht in gleicher Weise, wie beim Laborarzt erwartet, detailliert nachgewiesen werden. Als Beispiel für die Durchführbarkeit dieser Forderung sehen wir die Regelung der RöntgenWeiterbildung im Rahmen verschiedener Fachgebiete. Analog zu anderen Fachgebieten fordern wir von jedem Arzt, der Laboratoriumsuntersuchungen durchführt, hierfür eine qualifizierte Weiterbildung unter laborärztlicher Aufsicht, entsprechend den Zeiten laborärztlicher Weiterbildung." (Mit dieser Entsprechung ist die interne Weiterbildung des FL unter internistischer Aufsicht gemeint Die Red.)

Inzwischen wurden diese Vorstellungen vor allem bei dem Hauptgeschäftsführer der Kassenärztlichen Bundesvereinigung vorgetragen und unter Mitwirkung erfahrener Laborärzte ein Konzept erarbeitet, das den Forderungen des $\$ 368$ n (8) RVO ge- recht wird. Dieser Abschnitt erwähnt die Gemeinschaftseinrichtungen niedergelassener Ärzte (GEnÄ). Der volle Wortlaut der Vorstellungen wird im Februar-Heft dieser Zeitschrift veröffentlicht werden.

Ferner hat während der 11. Fortbildungsveranstaltung am 15 . November 1977 die Arbeitsgruppe zur Qualitätssicherung von Bindungsanalysen (zur Zeit vorwiegend Radioimmuno- und Enzymimmunoassay; Vorsitz Prof. Dr. med. G. Gries) eine Entschließung erarbeitet und für die Veröffentlichung vorbereitet. Sie hat folgenden Wortlaut:

„Die Deutsche Gesellschaft für Laboratoriumsmedizin hält es in Anbetracht der besonders hohen Verantwortung beim Uingang mit Bindungsanalysen und in der diagnostischen Wertung von damit gewonnenen Ergebnissen für unumgänglich, als zuständigé Fachgesellschaft Richtlinien zur Qualitätssicherung vorzulegen.

Mit den Bindungsanalysen, wie z. B. den Radioimmunoassay bzw. den Enzymimmunoassay, werden erheblich geringere Stoffmengen nachweisbar. Dadurch können Bestandteile menschlicher Körperflüssigkeiten mit hoher biologischer Wirksamkeit gemessen werden. z. B. Hormone, Araneimittel, Antikörper, Antigene und andere diagnostisch relevante Substanzen

\section{Eimbanddecken für den Jahrgang 1977 LABORATORIUMSMEDIZIN erhalten Sie zum Preis von DM 11,80 zuzüglich Porto und MWSt. direkt vom VERLAG KIRCHHEIM + CO GmbH Postfach 2524, 6500 Mainz \\ für die Zeitschrift}


: Nrehadiespmosrik usw. ). Die hohe Aussuget raft von linfersuchungsergeh.

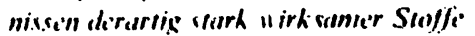
kamn hol Fohlhestinumunge"l schucerwiosendi Folgen fiur den Parienten haben.

Dic Sicherheit einer diagnesstischen

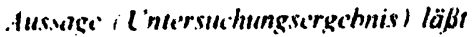
durch Steigernung wer Lintersuchungsrtualistït einc Kinstendämpfiing dhurch lirmintirumg der Lintersuchungszahl :II.

Dic Richlinien der Deutsche'n Gescllschati fïr Laboratoriumısmedizin le'men sich an dic Empfehlungen des HHO E.xpert Committee on Biological Standardization (Tw'enty-sixth Report, Techinical Report Series Nr. 565. Genf 1975)* an und ergänzen vordringlich die Forderungen hinsichtlich der internen und externen Qualitätskontrolle.

I'egen der besonderen Situation, die sich durch das KrankenversicherungsKostendänipfungsgesetz (KVKG) ergibt, haben die zuständigen ärztlichen Körperschaften und gegebenenfalls auch Behörden im Augenblick die Möglichkeit uild dadurch die Verantwortung, dafïr zu sorgen, daß yor Aufnahme neuer V'erfahren dieser Art in Leistungskataloge den Forderungen der Qualitätskontrolle nach den Richtlinien der Deutschen Gesellschaft fiir Laboratoriumsmedizin entsprochen "ird."

Herr Kley berichtete über die Diskussionsergebnisse auf der Tagung der Internationalen Atomenergie-Kommission (International Atomic Energy Agency) besondere Bedeutung. Sie tagte vom 31. Oktober bis 4. November 1977 in Berlin. Einen breiten Raum dieses Symposiums nahmen Vorträge, Roundtable-Gespräche und Diskussionen über die interne und externe Qualitätskontrolle der RIA ein. Von allen Fachleuten wurde einheitlich die Meinung vertreten, daß eine wesentliche Voraussetzung der Qualitätssicherung von Radioimmunoassays

* Siche Bericht im Dezember-Heft Seite XXI und den Beschluß der Vertreterversammlung der Kassenärztlichen Bundesvereinigung am 9. Mai 1977 (Juni-Heft Seite XXI). dic Stabilität des angewendeten Meß systems ist. Diese Stabilität ist vor. handen. wenn sich die Standardabweichungen der gemessenen lichkurvenwerte innerhalb eines vorgegebenen Dosiswirkungsprofiles bewegen (Präzisions- oder Fehlerprotile). Die Ubcrprüfung der Standardabweichungen der eingesetzten Präzisionskontrollen crfolgt ebenfalls am besten in Form solcher Präzisionsprofile. Wegen der häufig fehlenden Richtigkeitskontrollen bestehen zur Zeit mit Ausnahme der Steroid-RIA noch keine verbindlichen Hinweise für festgelegte Fehlergrenzen. Die interne Qualitätskontrolle einschließlich der Überprüfung der Systemstabilität dient kurzfristig zur kritischen Analyse der erzielten Ergebnisse und langfristig, z.B. über Erstellung von Präzisions- und Fehlerprofilen, zur Optimierung der Assay-Auslegung.

Ein einheitliches Verfahren zur Durchführung der externen Qualitätssicherung bei radioimmunologischen Untersuchungen konnte trotz intensiver Diskussion einzelner bisher durchgeführter Ringversuche von den Fachleuten nicht empfohlen werden. Durch die WHO wird zur Zeit ein Ringversuch zwischen 150 Laboratorien der ganzen Welt unter Verwendung sogenannter „Matched reagents" zur radioimmunologischen Bestimmung von Gonadotropinen und Sexualsteroidhormonen durchgeführt. Die ersten Teilergebnisse dieses interessanten Ringversuches zeigen, daß durch die Einführung einer einheitlichen Methode die Präzision und Richtigkeit der RIA-Meßresultate wesentlich verbessert werden können. Aus diesen Gründen haben Fachleute, wie Ekins, Rodbard, Hall und Jeffcoate, allen Ländern empfohlen, standardisierte RIA-Verfahren zur Optimierung und Vereinheitlichung der Meßresultate einzuführen. In Deutschland wird von Prof. Dr. med. P. C. Scriba und seinen Mitarbeitern, München, die durch eine eigene Form von ${ }^{\complement}$ RIA-Ringversuchen in den letzten Jahren besonders bekanntgeworden waren, 1978 erstmalig ein Ringversuich für TSH nach dem Vorbild der WHO-Ringversuche durchgeführt werden.
Die zukünftigen Entwicklungen auf Jem Gebiet der RIA-Qualitätskontrolle werden den Anwendern dic Möglichkeit geben, durch den Finsatz sogenannter ,.standardisierter“ Verfahren und homologer Kontrollproben vergleichbare Meßresultate $\pi$ erzielen.

Ein weiterer Gesprächskreis der 11. Fortbildungsveranstailtung der Laborärzte befaßte sich mit dem Thema: Verantwortungsbereiche von MTA, MTLA und Laborarzt (Vorsitz: Prof. Dr. med. H. Reinauer). Über dessen Ergebnisse wird in einem der nächsten Hefte berichtet werden.

Die Meinungsbildung zum Thema .SI-Einheiten" durch den AusschuB "Lehre und Wissenschaft" (Vorsitz: Prof. Dr. med. A. Rösler-Englhardt) und eine ausführliche Aussprache auf der Mitgliederversammlung ist bereits im Dezember 1977 in dieser Zeitschrift veröffentlicht worden. Sie stellt vor allem darauf $a b$, daß übereilte Entscheidungen nicht erforderlich sind, weil sich die Laboratoriumsbefunde schon seit längerer Zeit nach den Forderungen des SI-Systems richten, obgleich dies in der Medizin nicht gefordert ist.

Ein Gesprächskreis führte einen Gedankenaustausch durch über die Problematik von ,,Zulassung, Beteiligung, Ermächtigung zur kassenärztlichen Versorgung auf dem Gebiet der Laboratoriumsmedizin", (Vorsitz: Dr. med. O. Fenner), der im wesentlichen den Gesichtspunkt der Bedürfnisprüfung herausstellte und feststellte, daß die praktische Handhabung in beide Richtungen starke Unterschiede aufiveist. Auch die Änderung der RVO durch das KVKG dürfte nicht ganz ohne Einfluß auf diesen Fragenkomplex sein, wenngleich er expressis verbis fast nicht berührt wurde. Die Bestrebungen der Regierung, die Grenzen zwischen ambulanter Versorgung durch niedergelassene Ärzte und ambulanter Versorgung durch Krankenhausärzte zu überbrücken bzw. zu verwischen, dürften sich auf Dauer auch auf diesem Gebiet bemerkbar machen.

LK. 


\section{Aktuelle Labordiagnostik auf der Medica ' $77^{*}$}

\begin{abstract}
Über 11000 Ärzte und Angehörige der Heilhilfsberufe haben auf der Düsseldorfer Medica ' 77 in mehr als 300 Vorträgen, Seminaren und Kursen ihr Wissen über aktuelle Forschungsergebnisse aus Klinik und Praxis erweitern können. Speziell für Ärzte hat die Deutsche Gesellschaft für Laboratoriumsmedizin ein Symposium mit insgesamt fünf Rundtischgesprächen mit dem Titel „Laboratoriumsdiagnostik von Immunopathien" durchgeführt.
\end{abstract}

\section{Unter der Moderation von}

Drof. Dr. med. Anneliese RöslerEnglhardt, Zentrallaboratorium des Rudolf-Virchow-Krankenhauses Zerlin. sowie Prof. Dr. med. Wolf- gang Diefenthal, Institut für Experimentelle und Klinische Virologie am Berliner Wenckebach-Krankenhaus, entwickelte sich zwischen den wissenschaftlichen Referenten und dem ärztlichen Auditorium eine lebhafte Diskussion. Nur durch eine sehr straffe Leitung des Seminars war es möglich, die durchzuarbeitende Stoffülle in der knapp bemessenen Zeit von kaum vier Stunden zu bewältigen und dennoch die von den Zuhörern gestellten Fragen hinreichend $\mathrm{zu}$ beantworten.

Fragen der Immunhämatologie, insbesondere immunhämatologische Anämien, griff der Freiburger Wissenschaftler Prof. Dr. med. Helmut

Aktuelle Probleme der Laboratoriumsmedizin wurden auf der Medica '77 in Referaten Ind Diskussionen aufgegriffen. Auf dem Podium Teilnehmer der Rundtischgespräche luf dem Symposium ", Laboratoriumsdiagnostik von Immunopathien" am 17. Novemjer 1977. Von links nach rechts: Prof. Dr. H. Schubothe, Abteilung für Immunpatholosie der Medizinischen Universitätsklinik Freiburg; Prof. Dr. K. Federlin, Direktor der Med. Universitäts-Poliklinik Gießen; Prof.Dr. H. Warnatz, Institut für klinische Immunologie der Universitāt Erlangen-Nürnberg; Frau Priv.-Dozentin Dr. U. Rother, institut für Serologie und Immunologie der Universität Heidelberg; Prof. Dr. H.P. Seelig. Institut für Serologie und Immunologie der Universität Heidelberg.

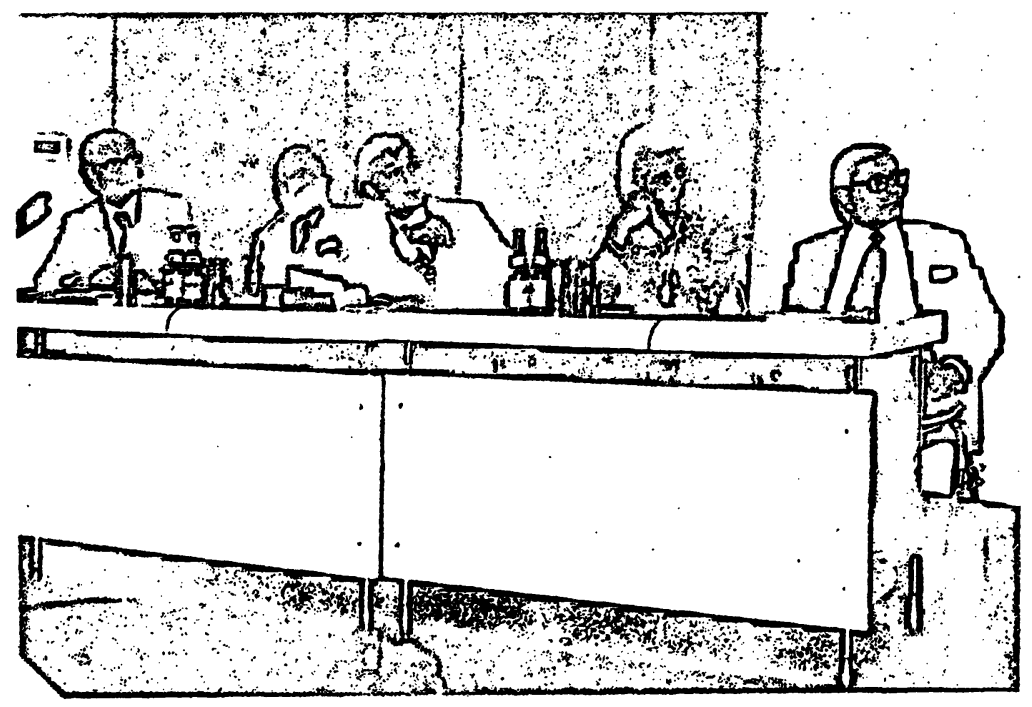

Schubothe in seinem Kurzreferat auf. Er ging dabei auf die Bedeutung von Wärmeautoantikörpern, Kälteagglutininen, komplementabhängigen Autohämolysinen sowie Antikörpern bei medikamentabhängigen immunhämolytischen Anämien ein. Außerdem schilderte er die Bestimmung und Bedeutung von Antikörpern bei Immunthrombopenien. Inhalt des zweiten Rundtischgespräches unter Leitung von Dr.med. H. W. Baenkler, Erlangen. war die Bedeutung der Immunglobulinspiegel und der Komplementspiegel, die Bestimmung und Bedeutung von $\mathrm{HB}_{\mathrm{s}}$-Antigen, $\mathrm{HB}_{\mathrm{c}}$-Antigen und e-Antigen und von Antikörpern bei akuten und chronischen Lebererkrankungen. Ein besonderer Diskussionspunkt war das Befundmuster humoraler Antikörper bei chronischen Leberkrankheiten und seine diagnostische Bedeutung.

„Immundiagnostik bei Nierenerkrankungen" war Thema des dritten Rundtischgespräches unter Leitung der Heidelberger Wissenschaftler Frau Priv.-Doz. Dr. Rother und Prof. H. P. Seelig. Angesprochen wurde dabei die Immundiagnostik an Nierenbiopsien und ihre praktische Bedeutung, der Nachweis von zirkulierenden Immunkomplexen und Veränderungen des Komplementspiegels bei den doppelseitigen hämatogenen Glomerulonephritiden.

Im Rahmen des Themas .,Immundiagnostik bei immunopathologischen Systemerkrankungen" (Kollagenkrankheiten) wurde auf die Bedeutung der Immunglobulinspiegel und Komplementspiegel in der Pra-

- Ein zusammenfassender Berichi üter den Inhalt der Vortrage erscheint in einem der năchiten Hefte der Zeir. schrifi LABCRATORIUMSAIIDIZIN im Teil WISSEN SCIIAFT UND FORTILILIOUNG. 
xis cingegangen. Dic L.citung des (iesprichs stand unter den Prolicssoren $K$. Federlin, (iicken, und H. Warnats. Erlangen. Zur Sprache kamen innerhalb dicses Komplexes Nachweis und Bedeutung von antinukleären Antikörpern, anti-DNSAntikörpern. Antikörpern gegen das etrahicrbare nukleäre Antigen (enA) und von antinitochondrialen Antikörpern. Dabei wurden auch Methoden zur Bestimmung der Rhcumafaktoren und ihre Wertigkeit erliatutert.

Abschließend wurde die Immundiagnostik bci Endokrinopathien behandelt, wobei es vor allem um die Erhebung immunologischer Befunde bei Schilddrüsenerkrankungen ging. Prof. Federlin ging dabei auf Nachweis und Bedeutung der Antikörper gegen Thyreoglobulin und gegen das mikrosomale Antigen der Schilddrüsenzelle ein und erläuterte Probleme im Zusammenhang mit dem long acting thyroid stimulator (LATS) und dem human specific thyroid stimulator (HTS). Im Rahmen der Immundiagnostik bei Diabetes wurde über den Nachweis von Allergien gegen Insulin diskutiert sowie über
Methoden rum Nachweis von Insulinantikörpern bei Insulinresistenz und den sich daraus ergebenden Konsequenzen.

Schon bei dem Versuch einer Kurzzusammenfassung des im Seminar angebotenen Stofles wird deutlich, welchen hohen wissenschaftlichen Standard diese Fortbildungsveranstaltung hatte. Die Bereitschaft der Ärttc zur freiwilligen Fortbildung lic $\beta$ sich an diesem schwierigen, aber hochaktuellen und bedeutenden Thema besonders gut messen.

Das Wort von Prof. Dr. med. Dr. h.c. Ernst Fromm, Präsident der Deutschen Gesellschaft zur Förderung der Medizinischen Diagnostik e.V. und Ehrenvorsitzender der Deutschen Gesellschaft für Laboratoriumsmedizin, zu dem Ergebnis der fünf Kongreß- und Ausstellungstage gilt auch für diese Veranstaltung: „,Der überragende Erfolg dieser 9. Medica in Düsseldorf zeigt, daß trotz aller gegenteiligen Meinung Ärzte und Angehörige der Heilhilfsberufe diese Form der freiwilligen Fortbildung mit großem Interesse in Anspruch nehmen. Dieser Aufwärts-

Auch zum Symposium der Deutschen Gesellschaft für Laboratoriumsmedizin auf der Düsseldorfer Medica kamen Mediziner aus dem gesamten Bundesgebiet.

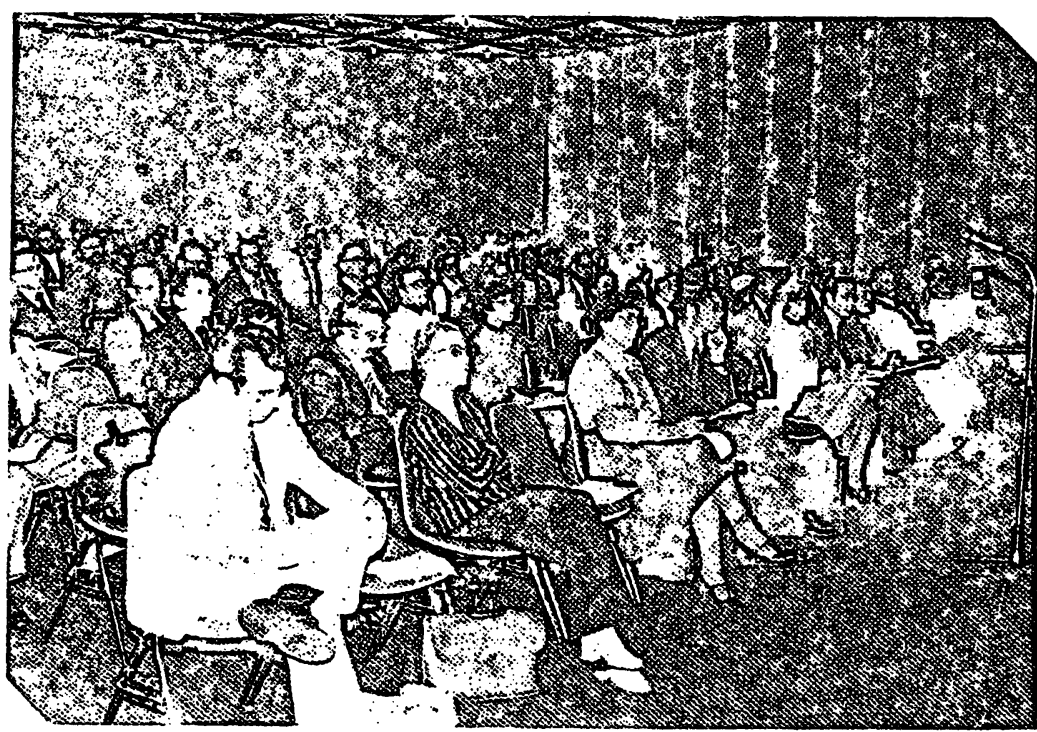

trend verpflichtet dazu. die Qualitāt der Fortbildung in Düsseldorf von Jahr zu Jahr zu steigern und das Programm auch künftig so zu gestalten, dab seine $\wedge k$ tualität dem Arzt in Praxis und Klinik unmittelbar zugute kommt."

Re.

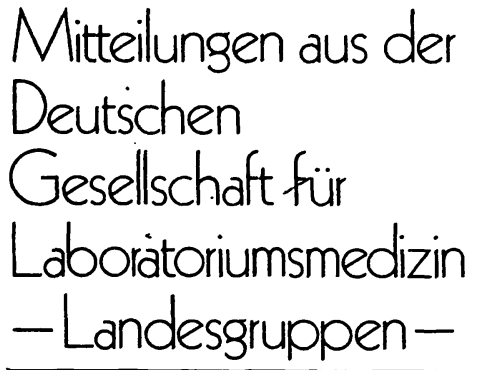

\section{Baden-Württemberg}

Im Land Baden-Württemberg, und zwar für alle Kammerbereiche dieses Bundeslandes, wurden durch Briefwahl gewählt :

Dr. med. Wolfgang Hauck, Karlsruhe. zum Landesobmann und Professor Dr. med: Hans Haug, Stuttgart, zum stellvertretenden Landesobmann.

Beide Kollegen sind über ihren örtlichen Wirkungsbereich hinaus bekannt àls engagierte Laborärzte, die sowohl in ihren fachlichen als auch in beruflichen Bereich hervortraten. Aulgrund der guten Kontakte mit der Bundesgeschäftsstelle und dem Vorstand ist eine wesentliche Intensivierun! der Tätigkeiten sowohl im Bereich von Wissenschaft und Fortbildung als auch auf dem beruflichen Gebiót zu erwarten. 


\section{Mitteilungen aus der Österreichischen Gesellschalt für medizinische und chemische Labordiagnostik*}

\section{Eine Fachgesellschaft stellt sich vor}

Bis Ende 1970 gab es in Österreich für dieses Fach keinen Facharzttitel. Jeder Arzt, der glaubte, die notwendige Eignung für den Betrieb eines medizinisch-diagnostischen Labors zu besitzen, stellte an den Obersten Sanitätsrat beim Bundesministerium für soziale Verwaltung ein entsprechendes Ansuchen und bekam, nach Überprüfung seiner Ausbildung, eine Konzession zum Betrieb eines Labors. Der Oberste Sanitätsrat hatte damals eine gewisse Normierung der Ausbildung seinen Entscheidungen zugrundegelegt.

So wurden im allgemeinen mindestens folgende Fächer verlangt:

1 Jahr Innere Medizin. 6 Monate Hämatologie, 3 Monate Serologie, 9 Monate Pathologie (davon möglichst 6 Monate Bakteriologie) und 2 Jahre Tätigkeit in einem Zentrallabor eines großen Krankenhauses oder in einem medizinisch-chemischen Institut. Dieser Spezialausbildung mußte eine abgeschlossene dreijährige Ausbildung zum praktischen Arzt oder eine Facharztausbildung, womöglich Innere Medizin. vorangegangen sein.

So war der Stand der, Ausbildung für • das Fach Labormedizin. als mit 1. Jänner 1971 der ,,Facharzt für medizinische und chemische Labordiagnostik" in die Ärzteausbildungsordnung aufgenommen wurde. Nachdem es einen Facharzt für die Laboratoriumsmedizin nun auch in Österreich gab und die Zahl solcher Fachärzte zunahm, wurde es notwendig. eine entsprechende Vertretung dieses Kollegenkreises zu schaffen. Wohl gab es in den Ärztekammern für Wien und Oberösterreich Fachgruppen für Laboratoriumsmedizin, aber in den restlichen 7 Bundesländern

\section{Der Vorstand}

der Österreichischen Gesellschaft für medizinische und chemische Labordiagnostik setzt sich wie folgt zusammen:

Präsident: Dr. med. Hans Lackner, Wien (zugleich Vorstandsmitglied der Österreichischen Gesellschaft für Klinische Chemie und Referent der Österreichischen Ärztekammer)

Vizepräsident: Dr. med. Paul Hübl, Wien

2. Vizepräsident: Dr. med. Friedrich Urban. Wien

Schriftführer und Redaktionskomitee: Dr. med. Hans Jörg Klein. Wien

Referent für wissenschaftliche Angelegenheiten: Prof. Dr. med. Franz Gabl. Wien (zugleich Vizepräsident der Österreichischen Gesellschaft für Klinische Chemie)

Finanzreferent: Dr. med. Franz. Jauk, Wien

Referent für Qualitätskontrolle: Dr. med. Heinrich Spitz, Wien

Auslandsreferent: Dr. med. Hans Georg Klein, Wien

Kechnungeprüfer sind: Dr. med. Kurn Mustafa. Wien. und Dr. med. Michael Rosah. Wien

waren die Laboratorien mehr oder weniger sich selbst überlassen. Der Grund dafür ist sicher in der wahlarithmetrischen Bedeutungslosigkeit einer so kleinen Gruppe und einem gewissen Neid der übrigen Kollegenschaft zu sehen, die nur die Umsätzc der diagnostischen Laboratorien, aber nicht deren hohe Kosten für apparative Ausstattung und den Betrieb sahen. So erschien es immer notwendiger, eine gemeinsame Vertretung aller Fachärzte für Labormedizin anzustreben. Nach vergeblichen Versuchen, im Rahmen der Österreichischen Ärztekammcr als drittes, sogenanntes technisches Fach. nach Radiologie und Zahnheilkunde, eine entsprechende Organisation aufzubauen, kam es im März 1973 zur Gründung der Österreichischen Gcsellschaft für medizinische und chemische Labordiagnostik. Der Titel ist nicht sehr eindeutig und gibt immer wieder Anlaß zu Verwechslungen. Er wurde jedoch gewählt, weil die offizielle Bezeichnung des Facharztes im Gesetz den gleichen Wortlaut trägt. Trotz der scheinbar eindeutigen Trennung von Medizin und Chemie durch das unscheinbare, aber in diesem Fall äußerst wichtige Wörtchen ,und" wird das Fach ständig mit der medizinischen Chemie verwechselt. Gründe hierfür sind sicher auch darin zu suchen, daß in den Laboratorien der großen Krankenanstalten oft die Serumchemie im Vordergrund steht und daß die übrigen Aufgaben der Laboratoriumsmedizin im Spital - regional sehr unterschiedlich - in den Hintergrund Ireten. In der freien Praxis macht jedoch dic Serumchemie kaum ein Drittel bis Vicrtel der Gesamttätigkeit cines Laborarztes aus, und

- Die Zeitschrift LABORA TORIUMSMI:DIZIN hált seil September 1977 auch dic Organuchati Jieser Scliwestergesellschali in Osterretich. 


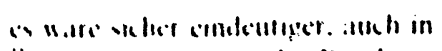

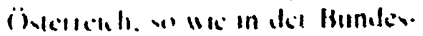

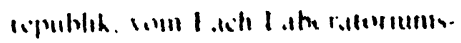

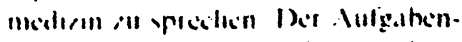

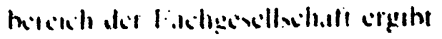
wh all dem ticilylen nun herells

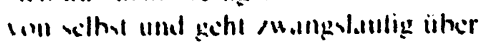

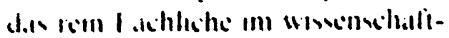
lichen sinn hinalus, $1: m$ dis Bild kedinh absurunden. ist on notwendig.

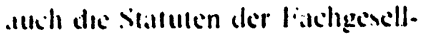
whill sl hemlen.

\section{Auszug aus der Satzung der Österreichischen Gesellschaft fïr medizinische und chemische Labordiagnostik}

\section{$\$$}

a) die "imenschaftlichen. fachlichen und heruflichen Belange der Labordiagnostik zu lördern.

b) laulend die wissenschaftlichen Grundlagen für die hestmögliche labordiagnostische l'ersorgung der österreichischen Bevölkerung zu erarbeiten.

c) die internationale Zusammenairbeit auf den Gebiete der Labordiagnostik zu pllegen.

Damit dient sic der Volksgesundheit, der $W$ issenschaft und der Völkerverständigung.

\section{$\$ 5$}

Der Verein hat

a) ordentliche Mitglieder

b) korrespondierende Mitglieder

c) Ehrenmitglieder

d) außerordentliche Mitglieder.

\section{\$10}

Organe des Vereins sind:
a) der Vorstand
b) die Mitgliederversammlung
c) die Rechnungsprüfer
d). das Schiedsgericht.

\section{§ 11}

Der Vorstand setzt sich aus 7 ordentlichen Mitgliedern zusammen:
a) der Präsident
b) zwei Vizepräsidenten
c) der Schriftü̈hrer
d) der Finanzreferent
e) zwei weitere ordentliche Mitglieder der Gesellschaft.

Die Amtsdauer der Vorstandsmitglieder und Rechnungsprüfer beträgt je fünf Jahre.

\section{Wissenschaftliche fortbildungstagung liir Laboraloriumsmedisin Ohargurgl Tirol. (i)sterrich vom 8. bis 16. April 1978}

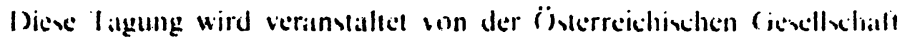
lïr medisinische und chemiscle labordiagnostih. gemeinsam mil der 1)eutschen (iescllschalf für lahoratoriumsmedizin. dem Rederall für labormedirin der (̈)serreichischen Ärstchammer und den schweter

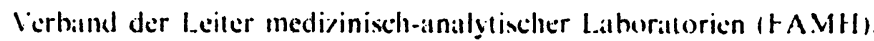

Dic (irundausrichtung der Veranstaltung soll die intensive medisinixche Dishussion und aktuelle Information außerhalb des üblichen Rahmen. der Klassischen KongreBatmosphäre sein. Der gewähltc Tagungsort Obergurgl bietel durch seine nattürliche Lage in $1950 \mathrm{~m}$ Sechöhe inmitten der herrlichen Bergwelt der Alpen dic Gewähi dafür. daß sich die Tagungsteilnehmer aus der hektischen Allagsumgebung lösen und der wissenschafllichen Weiterbildung widmen können. Die Tagung findet im Kungrebsatal des Hotels ..Hochtirst" statt. der etwa 250 Télnehmern Platz bictet.

Dic Hauptthemen sind

> Serumproteine

$>$ Gerinnungsdiagnostik

> Inzymimmunassay-Radioimmunassay

Dic Anmeldefrist lür Kurzrelerale von maximal (1) Minuten Dauer ist Ende Dezember 197? abgelaulen. Sicherheitshalber sollte fïr besonders interessante und moderne Mlitteilungen cine Verhindung versucht werden mit:

Österreichische Gesellschaft für medizinische und chemische Labordiagnostik. A-1010 Wien. Franz-.Josefs-Kai 65

Folgende Einzelthemen stehen bereits fest:

Serumproteine:

Klinische Bedeutung dor Immunelektrophorese

Frfahrung mit der turbidimetrischen Plasmaproteinbestimmung auf einem Zentrifugalanalysator

Immunglobulinbestimmung mit dem Laser-Nephelometer

Transferrin-EBK. ein Methodenvergleich

Immunglobulinbestimmung mit Analysenautomaten

Spezifische Plasmaproteine - eine klinische Übersicht

Gerinnungsdiagnostik:

Klinische Aussagekraft elektronischer Zählung und Größenbestimmung der Thrombozyten sowie der Plättchenaggregation

Diagnose der Verbrauchskoagulopathie

Photometrische Gerinnungsanałysen mit synthetischen Substraten

Enzymimmunassay-Radioimmunassay:

Vergleichende Untersuchungen zur Digoxinbestimmung im Serum mit ELISA. EMIT und RIA

Enzymimmunassiay zur Bestimmung von Antikonvulsiva

Drogen-Screening

Nachweis von Digoxin und seine Bedeutung

Bestimmung von alpha-1-Fetoprotein mit EIA

Verständlicherweisc ist die Teilnehmerzahl begrenzt und erfolgt deshalb in der Reihentolge der Voranmeldung. Diese ist unverbindlich. Erst nach Zusendung des endgültigen Programmes. der Korreșpondenz mit dem Veranstalter und der Einzahlung der Teilnchmergebühr wird die Anmeldung endgültig.

Obergurgl am Ende des Ötztales in Tirol ist erreichbar mit eigenem PKW' oder mit Linienbus bzw. Taxi ab Schnellzug-Station Ötztal aul der Strecke Bregenz-Feldkirch-Ariherg-Innsbruck. 


\section{Van-Swieten-Tagung 1977 und Laboratoriumsmedizin}

Auf der diesjährigen Van-SwietenTagung. dem österreichischen Ärztekongreß, fand am 26. Oktober 1977 wiederum die Jahrestagung der ..Österreichischen Gesellschaft für medizinische und chemische Labordiagnostik" statt, an der mehrere Vertreter der Deutschen Gesellschaft für Laboratoriumsmedizin, zugleich Arbeitsgemeinschaft der Fachärzte für Laboratoriumsmedizin e. V., teilnahmen. Der wissenschaftliche Teil dieser Tagung stand unter dem Motto ..Externe Qualitätskontrolle Effektivität von Ringversuchen", der von Prof. Dr. med. R. Merten, Düsseldorf, geleitet wurde und an der teilnahmen Dr. med. M. M. Müller. Wien, als Leiter der österreichischen Ringversuche und ferner die Leiter der Wiener Ringversuche, Dr. med. H. Spitz, Wien, sowie der Oberösterreichischen Ringversuche, Dr. med. R. Sommer, Linz, ferner der schweizerische Kollege Dr. M. Viollier, Basel.

Die Diskussion am runden Tisch ging über einen reinen Erfahrungsaus-

\section{Die Österreichische Gesellschaft}

für Klinische Chemie wählte auf ihrer Jahreshauptversammlung am 27. Oktober 1977 in Wien einen neuen Vorstand.

Präsident: Prim. Dr. med. Hans Jörg Gibitz, Leiter des Zentrallaboratoriums der Landesanstalten, Salzburg

Vizepräsident: Prof. Dr. med. Franz Gabl. Leiter des Zentrallaboratoriums der 1. Med. Universitätsklinik. Wien

Sekretär: Prim. Dr. med. Wolfgang Hohenwallner, Leiter des Zentrallaboratoriums des Krankenhauses der Barmherzigen Schwestern, Linz/Donau, Langgasse 16. Tel. 0043732/75181

Schriftruhrer: Prim. Dr. med. Peter Bayer, Leiter des Laboratoriums des Wilhelminenspitals. Wien

Kassier: Doz. Dr. med. Mathias Müller. Med.-Chem. Institut der Universität Wien. Wien

Beisitzer: Prof. Dr. med. Erwin Deutsch. Vorstand der I. Med. Univ. Klinik, Wien Prof.Dr.med. Erich Kaiser. Vorstand des Med.-Chem. Institutes der Univ. Wien Frau Dr. med. Dorothea Pastner, Med. Univ. Klinik, Innsbruck

Prof. Dr. med. Michael Fischer. Leiter des Laboratoriums des Lainzerkrankenhauses. Wien

Dr. med. Hans Lackner. Laborarzı Wien

tausch weit hinaus und wies die Wege für die zukünftige Entwicklung und die Möglichkeiten einer Zusammenarbeit. Hierüber wird im einzelnen noch an anderer Stelle berichtet.

Während dieser Tagung fand am 27. Oktober 1977 eine gemeinsame Vorstandssitzung der Österreichischen Gesellschaft für Klinische Chemie, der Österreichischen Gesellschaft für medizinische und chemische Labordiagnostik, der Deutschen Gesellschaft für Laboratoriumsmedizin. zugleich Arbeitsgemeinschaft der Fachärzte für Laboratoriumsmedizin $\mathrm{e} . \mathrm{V}$. und einem Vertreter der Schweizerischen Fachgesellschaft (Präsident ab 1. Januar 1978 Herr Dr. med. MarcAndrè, Basel) statt. Auf dieser Sitzung wurden Fragen der Förderung und Verbreitung dieser Zeitschrift LABORATORIUMSMEDIZIN behandelt, die seit einigen Monaten auch das offizielle Organ der österreichischen Fachgesellschaft ist. Dabei wurde unter anderem beschlossen, gegenseitig wichtige Gesetzestexte und andere für die Laboratoriumsmedizin bedeutsame Schriften durch Abdruck bekanntzumachen. Ferner diente die Sitzung der weiteren Vorbereitung des gemeinsamen Kongresses in Obergurgl im April 1978, weil in diesem Jahr der Deutsche Kongreß für Laboratoriumsmedizin nicht stattfinden wird. Dic Veranstaltung in den Ötztaler Alpen der österreīchischen Kollegen wird von den schweizerischen und deutschen Gesellschaften unterstülzt. Wie zu erfahren ist, ist diese Kongreßveranstaltung bereits fast vollständig ausgebucht. Ausführliche Ankündigung auf Seite $A B$ 10. Interessenten wenden sich baldmöglichst an die Geschäftsstelle der Deutschen Gesellschaft für Laboratoriumsmedizin in Leverkusen. Telefon 02172/74444, und erbitten dort Bestellkarten. 


\section{Aus wissenschaftlichen Gesellschaften und internationalen Gremien}

\section{Deutsche Gesellschaft \\ fiir Allergie- und Immunitätsforschung}

Dic deutsclic Gescllschaft für Allergic- und Immunitätsforschung schrcibt für 1978 den KarlHansen-Gedichtnis-Preis aus. Der Preis soll in erster Linie für eine Arbeit verliehen werden. die die Bedeutung der Forschung auf dem Gebiet der Allergic und Inmunologie hervorhebt. Arbeiten auf dem Gebiet der Allergie- und Inmmunitätsforschung sollen baldigst an den Schriftführer der Deutschen Gesellschaft für Allergie- und Immunitätsforschung, Prof. Dr. D. Ricken. Medizinische Klinik des St. JosefHospitals. 4630 Bochum, Gudrunstr. 56, gesandt werden.

\section{Deutsche Gesellschaft für Klinische Chemie}

Bereits 1970 hat dic Gesellschaft eine Kommission gebildet. die sich mit der Ausarbeitung von Empfehlungen zur Durchführung von klinisch-chemischen Untersuchungen bei der Prüfung von Arzneimitteln befassen sollte. Diese Kommission hat 1976 die „Empfehlungen der Deutschen Gesellschaft für Klinische Chemie zur Durchführung klinischchemischer Untersuchungen bei der Prüfung von Arzneimitteln" veröffentlicht (J. Clin. Chem. Clin. Biochem. 14, 161-164, 1976). Mitglieder der Kommission waren:

J. Breuer, Gelsenkirchen;

A. Delbrück, Hannover;

E. Gerhards, Berlin; P. Hajdu,

Frankfurt-Hoechst; W. Rick, Düsseldorf; L. Róka, Gießen;

K. Rommel, Ulm; D. Stamm, München; H. P. Wolf, Darmstadt. Im darauffolgenden Jahr wurden Kritik und Anregungen durch Gespräche mit dem Geschäftsführer des Bundesverbands der pharmazeutischen Industrie, Herrn Marris, der Leiterin der Sektion Klinische Pharmakologie der Deutschen Gesellschaft für Pharmakologie, Frau Weber, sowie Herren des Instituts für Arzneimittel des Bundesgesundheitsamts gesammelt. Ende des Jahres soll die überarbeitete 2. Empfehlung in der selben Zeitschrift veröffentlicht werden.

Anhang

Empfehlungen der Deutschen Gesellschaft für Klinische Chemie zur Durchfuihrung klinischchemischer Untersuchungen bei der Prüfung von Arzneimitteln

Die in der Bundesrepublik Deutschland von Fachsereinigungen bereits vor längerer Zeit veröffentlichten Empfehlungen zur Arzneimittelprüfung beziehen sich nicht auf den Bereich klinisch-chemischer Untersuchungen [Mitteilung des Vorstandes der Deutschen Pharmakologischen Gesellschaft und der Kommission zur Aufstellung von Richtlinien für die Prüfung neuer Arzneimittel. NaunynSchmiedeberg's Arch. Exp. Pathol. Pharmakol. 245 (1963) Anhang S. 20 und Mitteilungen des Vorstandes der Deutschen Gesellschaft für Innere Medizin zur Aufstellung von Richtlinien für die klinische Prüfung von Arzneimitteln. z: B. Klin. Wochenschr. 43, 698 1965)]. Da auf Grund der nur bedingten Übertragbarkeit tierexperimentell ermittelter Befunde auf den Menschen eine sehr sorgfältige Planung auch bezüglich der Durchführung klinisch-chemischer Untersuchungen unerläßlich ist, hat die Deutsche Gesellschaft für Klinische Chemie die bei der Prüfung von Arzneimitteln gesammelten Erfahrungen zusammengefa $B t$. Mit den verfaßten Richtlinien soll auch gewährleistet werden, daß Genauigkeit und Aussagekraft der Ergebnisse verschiedener Untersucher vergleichbar sind. Dic Richtlinien stellen ein Programm zur klinisch-chemischen Untersuchung von Körperfunktionen dar. Für die Prüfung vieler Arzneimittel wird es erforderlich sein, zusätzliche Untersuchungen vorzunehmen. Einschränkungen können in einer späteren Prüfphase in Einzelfällen sinnvoll sein (z. B. topische Anwendung).

\section{3. ordentliche Tagung eines Arbeitskreises „Mykobakterien" in Schüren, St. Ingbert, 27. 11. bis 28. 11. 1977}

Anlcinung zur Aufhereitung von Patienlemurin fïr alen Nadm cis rom Tulurkelhakterien*

\section{Materialsammlung:}

Nach Einschränkung der Flüssigkeitszufuhr am Vorabend soll der Morgenurin unter Vermeidung von Verunreinigungen in einem sterilen Gefä $B$ aufgefangen und so ins Labor gebracht werden.

Nach Möglichkeit soll der gesamte Morgenurin verarbeitet werden, mindestens aber $100 \mathrm{ml}$.

Für den Nachweis von Mykobakterien sind wenigstens drei Proben erforderlich, die an verschiedenen Tagen entnommen werden müssen.

\section{Anreicherung der Bakterien im Urin:}

Die Erfahrung hat gezeigt, da $\beta$ sich durch Zentrifugieren allein keine befriedigende Anreicherung der Bakterien im Urinsediment erreichen läßt. Deshalb sollte dem Zentrifugieren eine Eiweißfällung vorausgehen, bei der die Tuberkelbakterien mitgefällt und so am Grund des Gefäßes angereichert werden.

Dabei geht man in folgender Weise vor: Reaktionsgefäß: ein $200 \mathrm{ml}$ fassendes,

* Diese Anleitung wurde während der 3. ordentlichen Tagung eines Arbeitskreises „Mykobakterien" in Schüren/St. Ingbert vom 27. 11. bis zum 28. 11. 1977 erarbeitet. Dem Arbeitskreis gehören folgende Mitglieder an :

Professor Bartmann (Aprath), Frau Dr. Fink (Ruppertshain), Dr. Hussels (Berlin), Frau Dr. Jakschik (Frankfurt), Frau Dr. Müller (Schiffweiler), Professor Petersen (Gauting). Frau Dr. Roester (Schillerhöhe), Frau Dr. Schmidt (Würzburg). Dr. Schriever (Essen), Dr. Schröder (Borstel), Dr. Urbanczik (Schömberg),

Für die Unterstützung der Tagung danken wir der Saarstickstoff-Fatol GmbH, Schiffiveiler. 
schmales und hohes Glasgefäß (z.B. Meßzylinder), mit Alufolie abgedeckt und sterilisiert. Zu etwa $100 \mathrm{ml}$ Urin $1 \mathrm{ml}$ Serum oder Plasma (z. B. Reste von Untersuchungsproben aus der Med. Chemie) geben und sorgfältig durch Umschwenken mischen. Anschließend werden 5 Tropfen 20\%ige Sulfosalicylsäure (Merck Nr. 692 oder aus Merck 690 selbst eine 20\%ige Lösung herstellen) zugegeben. Danach nicht mehr umschwenken, sondern die sich bildende Fällung 2 Stunden bei $4 \pm 2{ }^{\circ} \mathrm{C}$ sedimentieren lassen. Der Überstand wird ohne Aufwirbeln bis auf einen Rest von 10-20 ml abgegossen. Dieser Rest kann durch Zentrifugieren (20 min bei $2500 \mathrm{~g}$ ) weiter eingeengt oder direkt der Vorbehandlung für die Kultur und/ oder den Tierversuch zugeführt werden.

Achtung: alle verwendeten Gefäße und die verworfenen Urinanteile müssen desinfiziert werden!

\section{Versand:}

Wenn die Tb.-Kultur und der Tierversuch nicht im gleichen Haus angelegt werden, muß der Versand des Urinsediments in Gefäßen erfolgen, die den Anforderungen des Deutschen Instituts für Normung e.V., Berlin (DIN 55515 Teil 1 Mrz 1977) entsprechen.

Sonderdrucke können bei Frau Dr. Roester. Fachklinik Schillerhöhe der LVA Württemberg. 7016 Gerlingen 2. angefordert werden.

\section{Leserbriefe}

\section{„Neue Versandpackungs- normen erhöhen \\ Sicherheitsanspriiche"}

Mit Erstaunen las ich in Ihrem o. g. Artikel in Heft $3 / 4$, Seite XVIII, daß die Richtlinien der Postordnung vom 31. November 1917 den neugenormten Versandpackungsnormen DIN 55515 , Teil 1 und 2. nicht entgegenstehen sollen.

Richtig ist jedoch, daß zur Zeit noch diese Ordnung gültig ist und damit jede andere Verpackungskombination außer Glas, Blech, Holz offiziell noch nicht zugelassen ist. Erst nach Änderung dieser mittlerweile 60 Jahre alten Verordnung können andere Verpackungsgefäße und -mittel zur Versendung von Untersuchungsproben verwendet werden.

Zwar hat die Post auf Grund einer Versuchsreihe der Firma Greinẹr offiziell bestätigt. daß die von der Firma entwickelten Kunststoffgefäße keinen Grund zur Beanstandung geben: Dieses ist jedoch nur für solche Ver- sendungsarten zugestanden worden, die „Flüssigkeiten ohne Krankheitserreger" enthalten. Damit können die neuen Verpackungsnormen zur Zeit offiziell ohne die Möglichkeiten eventueller rechtlicher Konsequenzen noch nicht angewendet werden. Trotzdem gewinnt der Leser des o. g. Artikels den Eindruck, daß diese neuen Verpackungsmaterialien bereits von der Post genehmigt wären.

Dr. R. Kley

Manforter Straße 225

5090 Leverkusen 1

\section{Persönliches'
Ehrung von Professor
Dr. med. Heinz Seeliger Dr. med. Heinz Seeliger}

Professor Dr. med. Heinz Sceliger, Leiter des Institutes für Hygiene und Mikrobiologie der Universitäı Würzburg, wurde in Anerkennung seiner Verdienste um die ärztliche Tätigkeit in der Laboratoriumsmedizin die Elrrenmitgliedschaft der Deutschen Gesellschaft für Laboratoriumsmedizin. zugleich Arbeitsgemeinschaft der Fachärzte für Laboratoriumsmedizin e. V.. durch Beschluß der Mitgliederversammlung am 14. November 1977 verliehen.

Professor Seeliger gehört zu den wenigen Wissen-

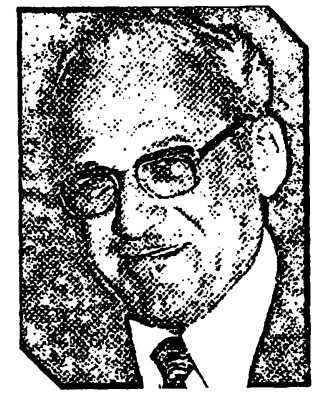
schaftlern, die seit den frühen Jahren des Bestehens des Berufsierbandes der Laborärzte. der Arbeitsgemeinschaft der Laborärzte Deutschlands, regen Anteil an den Problemen dieser damals noch kleinen Gemeinschaft genommen hat und insbesondere sein Spezialwissen sowie seine groBen Erfahrungen auf den mikrobiologischen Gebieten für die Fortbildung zur Verfügung stellie. In lebhafter Erinnerung ist den Teilnehmern jene Sonderveranstaltung ..Listeriose" während. des Kongresses für Laboratoriumsmedizin 1963 in Frciburg i.Br., die von Professor Seeliger zu einem einmaligen Erlebnis und zu einem zukunfisweisenden Programm gestaltet wurde. In vollendeter Abrundung. die nur dem überlegenen Kenner der gesamten Materie möglich ist, wurden alle Fragen der Bakteriologie, Serologie, Epidemiologie, Klinik und Therapie unter Einbezug des praktischen Vorgehens und der erforderlichen Übungen sowie Anschauungen abgehandelt. Damit hat Professor Seeliger für den Berufsverband der Laborärzte eine bedeutsame Entwicklung auf dem Gebiet der Fortbildung eingeleitet.

Der Geehrte war stets ein Berater in fachlichen und beruflichen Belangen, die aus sciner Universitätstätigkeit und auch scinen vielen internationalen Aufgabengebieten resultierte. Ihm wurden nicht ohne Grund das Aml des Präsidenten der International Association of Microbiological Societies übertragen, die ErnstRodenwaldt-Medaille in Gold 1975 in Anerkennung seiner Verdienste um die Planung und Errichtung des Nationalen Hygiene-Institutes in Lomé/Togo verliehen und andere internationalc Ehrungen angetragen.

Die wichtige Verbindung zwischen Ausbildungs- und Lehrstätle, Forschung und Wissenschaft einerseits und dem Berufsverband, der Arbeitsgemeinschaft der Laborärzte, andererșeits hat Professor Seeliger ebenso wic scin Lehrer Professor Habs stets lebendig gehalten. $1 \mathrm{hm}$ hat immer an einer guten Synthese zwischen Anforderung aus der Wissenschaft und Realisierbarkcit in der P'raxis des LatboAnforderung aus der Wissenschant und Realisierbarkcit in der Praxis des Latho-
ratoriums gelegen. 


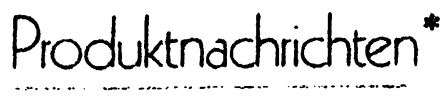

\section{Diagnosemikroskop fir dic Gynäkologic}

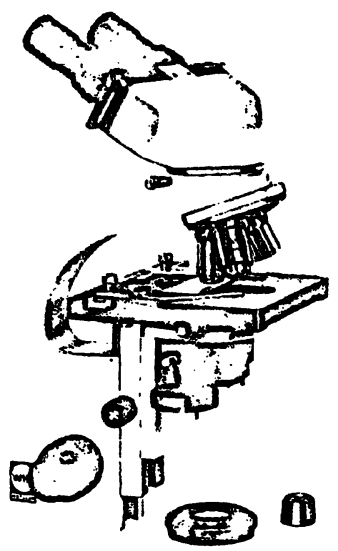

Dieses Labormikroskop ist speziell für die gynäkologische Schnelldiagnose von Vaginalabstrichen im Phasenkontrast konzipiert. Es wird damit eine komplette Geräte-Einheit geboten, bei der keine Einstellungen und Justierungen der Beleuchtungsoptik in herkömmlicher Art für die Phasenkontrastmikroskopie und das Arbeiten im Dunkelfeld erforderlich sind. Diese Bedienungsvereinfachung bringt Sicherheit für die optische Einstellung. womit eine schnellere Diagnose bei optimaler Bildqualität gewährleistet ist.

Mit dem Vergrößerungsbereich der beiden Objektive $\mathrm{Ph} 20: 1$ und $\mathrm{Ph}$ 40:1 sind außerdem ideale Voraussetzungen für zytologische Untersuchungen im Hellfeld, Dunkelfeld oder Phasenkontrast gegeben.

Für das Arbeiten im Phasenkontrast wird einfach der seitliche Wechselschieber mit der Ringblende am Kondensor eingeschoben und der Kondensor mit dem Triebaggregat bis zum Anschlag angehoben. Die Übereinstimmung der Phasenringe ist dann automatisch gegeben. Für Beobachtungen im Hellfeld wird der Wechselschieber nach außen gezogen, womit freier Lichtdurchgang gegeben ist. Für Untersuchungen im Dunkelfeld bleibt der Schieber mit dem Phasenring am Kondensor nach außen gezogen und lediglich die unten am Kondensor befestigte

* Die unter ,.Produktnachrichten“ wiedergegebenen Informationen beruhen auf Material, das die Firmen zur Verfügung gestellt haben. Die Angaben erscheinen somit außerhalb der Verantwortung der Schriftleitung.

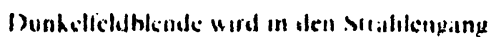
cimpenchuenkt.

Dils Mlikroshen Vh 36,5 Wilozyt wet romit ein uniserselles I.ahormikronkup rur Schnell-

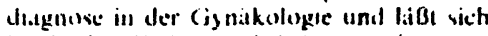
in gletcher Weice als Arbcilugeral fur /yto. luginche und hämalologisclie Untersuchunfen cinsetzen.

Nialuere Informationen üher Will Wetalar K(i, Postlach 40, 6331 Nauborn! Wetzlar.

\section{Bakteriologisches Material im Postversand}

Die Bundespost hat für den Versand von infektiösem Material, mit dem der Arzt im Laboratorium arbeiten muls, genaue Vorschriften erlassen. Nicht alles, was an Versandgefïßen angeboten wird, entspricht diesen Bestimmungen. Zusammengefaßt wird gefordert:

Feuchtes oder flüssiges Material ist in ein Glas mit Korkstöpselverschluß zu bringen. Dieses Gefäß ist in einem Blechbehälter zu verpacken. Zum Schutz und um austretende Feuchtigkeit abzufangen, ist aufsaugendes Material beizulegen. Der Blechbehälter wird in einem mit Deckel verschließbaren Holzkasten verpackt.

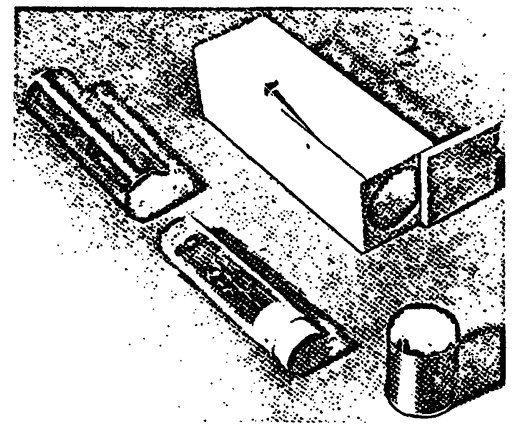

Das Kästchen ist mit einem roten Aufkleber „Vorsicht! Infektiöses Material!"” zu versehen. Der Versandbeutel soll genügend gro $B$ sein, so $d a \dot{B}$ sich die Sendung bequem abstempeln läßt. Auf dem Beutel ist der Vermerk „Vorsicht! Menschliche Untersuchungsstoffe!"” anzubringen.

Bei Verdacht auf Cholera, Pest, Tularaemie oder Rotz muß die Sendung per Schnellpaket erfolgen und ist dem Empfänger telegraphisch zu avisieren. Der Empfänger hat umgekehrt den Erhalt zu bestätigen.

Soweit auszugsiweise die noch heute voll gültige Postordnung aus dem Jahre 1917.

Bisher ist der ..Holzschachtel-Erla $\beta^{\prime \prime}$ voll gültig. Wer sich nicht dran hält - das gilt auch für staatliche Institute - verstößt eindeutig gegen die Postordnung. Allerdings, neue Gesetze werden kommen. Grundlage dazu dürfte die jetzt verabschiedete DIN 55515 sein. Ein Hinweis vom Gesundheits- ministerium: Die geltenden Beslimmungen sund hiv gur Neuregelung eunsuhalien. I Siche l.everhrief auf der Vorvecile () Kedl I

Ir ratanlich w: 1) Ilol/xhachtel int coner Kunsistoff.Verpachung in der stoblewtigkel! überlegen. Holkkäiven lasien wh audem nach (jebrauch leicht autoklavieren und ssmit nahezu beliebig oft wiederveruenden. Duss macht diese klassische Verpackung zu den preisgünstigsten Versindyetäben. Kunststolfgefïle und Plastikumhüllungen dagegen schmelzen bei flitzesterilisation zusammen; sie bleiben kostspielige tinuegartikel. die Müllkästen und Limwelt belasten.

Nähere Einzelheiten zu den Versandgefäßsen üher: SüBe \& Schmidt KG. Postfach 3140. 3502 Vellmar 3.

\section{Exakte Volumen mit der Ultipette}

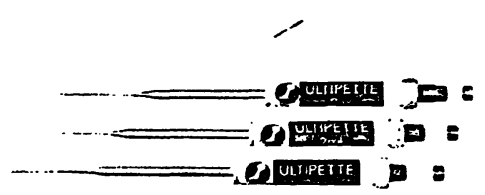

Die Ultipette ist ein einfacher Pipettiergriff für Pasteur-Pipetten. Das gewünschte Volumen (bis $2 \mathrm{ml}$ ) wird durch eine Schraube fest am Handgriff eingestellt. Die Glaspipetten werden in der Halterung durch einen Silikongummiring sanft, aber fest gehalten. Man braucht sie nur einzudrücken. und schon kann man das gewünschte Volumen pipettieren.

Der Pipettiergriff ist sauber, schnell und vor allen Dingen sicher. Es gibt keinen oralen Kontakt mit dem zu pipettierenden Medium mehr. Die Pipette eignet sich für fast alle gebräuchlichen Pasteur-Pipetten von einem Durchmesser von 6-8 mm. Der Preis beträgt ohne Pipetten DM 77.80 zuzüglich Mehrwertsteuer.

Lieferanschrift: Werner Zinsser, 6000 Frankfurt an Main 50, Postfach 501151.

\section{Schuittelgerät DSG 304 - universell einsetzbar}

Das universell einsetzbare Schüttelgerät DSG 304 ist mit vier leicht auswechselbaren Aufnahmetellern ausgestattet und eignet sich deshalb für Reagenzgläser, Flaschen, Kolben, Reagenzglasgestelle (bis zu 60 Reagenzgläser), Microtiter ${ }^{\mathrm{f}}$-Platten und Dosen. Die Schüttelfrequenz ist stufenlos regelbar (von $0-23 \mathrm{~Hz}$ ) bei einer Schüttelamplitude von $3 \mathrm{~mm}$.

Die Palette der Schüttelgeräté, auf die der Hersteller neben anderen Produkten des 


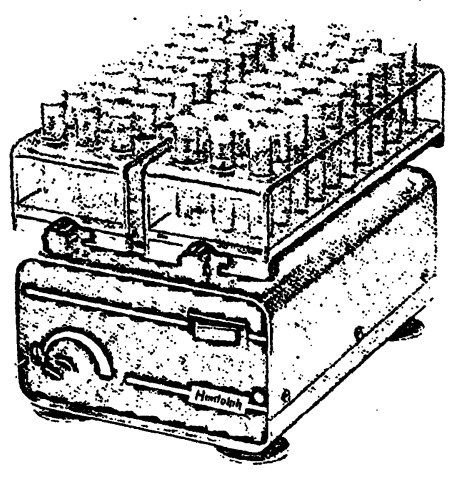

Laborbedarfs spezialisiert ist, umfaßt eine Reihe weiterer Modelle zum Durchmischen chemischer Substanzen.

Informationen über: Heidolph-Elektro KG, $\$ 420$ Kelheim.

\section{Sedimat-System zur schnellen Blutsenkung}

Mit dem Sedimat-System wird die Blutsenkung schnell, einfach, hygienisch und ohne jede Infektionsgefahr durchgeführt.

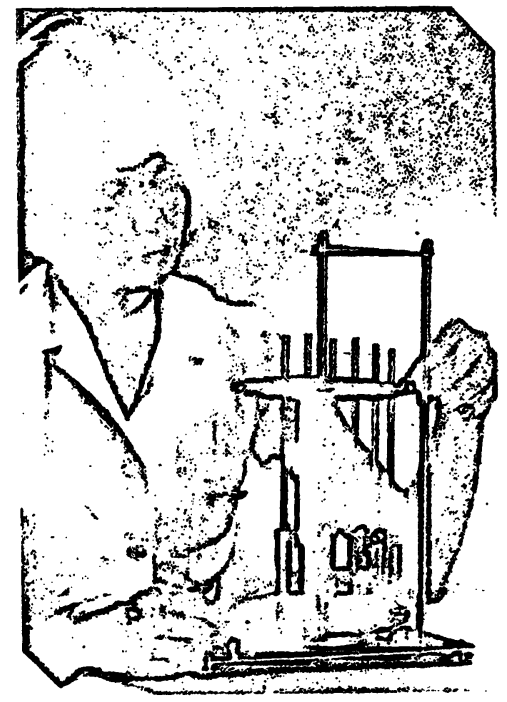

Die Sedimat-A usrüstung wird als komplettes System geliefert: 1) Sedimat-Gerät, 2) Polyäthylen-Behälter für die Aufnahme der Blutproben. 3) Polyäthylen-Abdichtung mit vorperforierter Markierung. 4) Westergren- $\mathrm{Pi}$ petten.

L.ieferung durch Müller ratiolab, Postfach 1, 6079 Buchschlag.

\section{Photometer mit UV- sichtbarem Spektralbereich}

Beckman Instruments stellt mit den Modellen 34/35 zwei neue Spektralphotometer für den UV-sichtbaren Spektralbereich vor. Die neuen Modelle erweitern die Serie der Spektralphotometer 24/25 und bieten: Messung bis 3 Extinktionseinheiten. verschiedene Zeitkonstanten, Vorschubgeschwindigkeiten bis $300 \mathrm{~nm} / \mathrm{min}$, einen low absorbance trigger sowie eine hellere, besser ablesbare Digitalanzeige.

Bewährte Baugruppen aus der Serie 24/25. wie das Schwingspiegel-System, die beiden

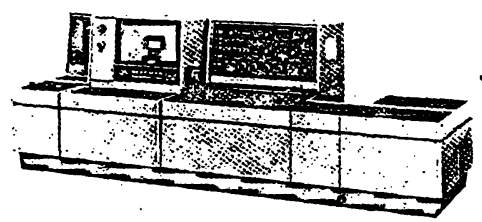

Spaltprogramme des Modells 25, die hervorragenden Mikromeßeigenschaften $(80 \mu \mathrm{l}$ Zellvolumen) sowie die Drucktastenbedienung wurden beibehalten.

Durch das umfangreiche Zubehörprogramm, das Angebot reicht vom temperierbaren Küvettenhalter über Proben- oder Küvettenwechsler bis zur Ulbricht-Kugel, wird für jedes Analysenproblem eine Lösung angeboten.

Informationen: Beckman Instruments GmbH, Frankfurter Ring 115, 8000 München 40 .

\section{Haematron 26 löst Zählprobleme im Labor}

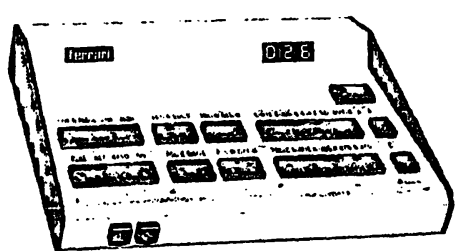

Das „Haematron 26" wird als universell einsetzbares Zählgeräl. angeboten. Die Differenzierung von Blutausstrichen, Knochenmarkpunktaten, dic Zählung von Thrombocyten und Retikulocyten läßt sich mit dem mil einem vollelektronischen Microcompu. tersystem ausgerüsteten Gerät bei bedienerfreundlicher Handhabung durchführen.

Herstcller: Ferrari electronic GmbH, Kurfürstendamm 175, 1000 Berlin 15.

\section{Gute Nachweisgrenzen mit JY 3-Spektralfluorometer}

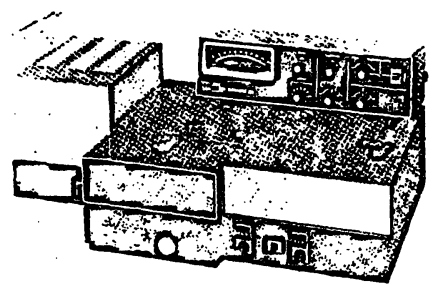

Das JY 3 Spektralfluorometer erreicht durch seine Hochleistungsoptik gute Leistungen. Da nur minimale Reflexionsverluste im Monochromator auftreten, kommt man zu ausgezeichneten Nachweisgrenzen.

Das Zweistrahl-Ratioverfahren garantiert Drifufreiheit bei Langzeitmessungen wie $z . B$. der Flüssigchromatographie. Hier bewährt sich auch die Durchflußeinrichtung mi Küvetten bis $8 \mu$ l Volumen. Das lästige, vom Lichtbogen der Lampe verursachte Rauschen in den meisten Fluorometern wird durch den fest eingebauten elektromagnetischen Lampenstabilisator beseitigt. Auch dies trägt zur Steigerung der Nachweisgrenze bei, da man ungestraft das Meßsignal mehr verstärken kann.

Eine Sonderausführung des JY 3 Spektralfluorometers ist für Messungen mit VidioQuantenzählern vorgesehen.

Service und Vertrieb durch: Instruments S.A. GmbH, Truderinger Str. 343, 8000 München 8 ?.

\section{Zellulose zur Ionenaustausch- chromatographie}

Für die Zellulose-Ionenaustauschchromatographie wird eine Zellulose, durch ein besonderes Verfahren in Perlform hergestellt. angeboten. Gute Durchflußeigenschaften. hohe Aufschlußgrenze, breite Gesamtionenkapazität, Sterilisierbarkeit. Volumenkunslanz sind cinige wichtige Eigenschaften dicses Produktes.

Informationen: Deutsche Pharmacia Gmbl. Munzinger Stratse 9. 78 Freiburg 1. 